\title{
Adolescent idiopathic scoliosis: an in depth analysis and historical review
}

\begin{abstract}
Adolescent idiopathic Scoliosis (AIS) is a common disorder of children about which there is a vast amount of research available for review. This manuscript aims to discuss significant classic and modern literature regarding this spinal deformity. An organized review of topics including the definition, epidemiology, etiology, physiology, diagnosis, and management of adolescent idiopathic scoliosis has been included herein. In this review, it is first explained that AIS now falls under the definition of late-onset idiopathic scoliosis. Epidemiologic discussion then reveals the relatively high prevalence of this disorder in children and lists the variations seen in prevalence by gender. Etiologic review explores the multifactorial nature of AIS and describes various conditions implicated in AIS pathogenesis including abnormalities in connective tissue structure, neuro-osseous development, platelet chemistry and hormone levels. The discussion of physiology explains the three dimensional structure of the AIS deformity and reviews long term complications such as back pain and compromised self-image. The section covering diagnostic methods highlights the physical exam for AIS and details important radiographic measurements including the Cobb angle, clavicle angle, and sagittal balance. Finally, the review of management emphasizes the importance of individualized therapy for AIS and discusses techniques involved in the non-operative and operative treatment of this spinal deformity.
\end{abstract}

Volume 3 Issue 4 - 2015

\author{
Jonathan R Zadeh,' Michael A Gleiber² \\ 'Florida Atlantic University, USA \\ ${ }^{2}$ Department of Biomedical Sciences and Spine Surgery, Florida \\ Atlantic University, USA
}

Correspondence: Michael A Gleiber, Florida Atlantic University College of Medicine, 1555 Palm Beach Lakes Blvd. STE 950 West Palm Beach FL 3340 I USA, Tel 56I-972-6464, Email admin@michaelgleibermd.com

Received: October 19, 2015 | Published: November 04, 2015

Keywords: Adolescent Idiopathic Scoliosis, Late-onset Idiopathic Scoliosis, Spinal Arthrodesis

Abbreviations: AIS, Adolescent Idiopathic Scoliosis, LIS, Late-onset Idiopathic Scoliosis

\section{Background}

Scoliosis, by its most basic definition, is a musculoskeletal deformity of the spine characterized by an observable lateral curvature. ${ }^{1}$ The diagnosis known as Adolescent Idiopathic Scoliosis (AIS) was first defined by John James in 1954 as he attempted to divide childhood idiopathic scoliosis into three peak periods of incidence. Adolescent onset was described as the appearance of scoliosis from the age of ten years until the end of growth, juvenile onset was described as appearance from the age of five to eight years, and infantile onset was described as appearance before the age of three years. ${ }^{2}$

More recent definitions of childhood scoliosis divide the pathology into two categories; early-onset, appearing before the age of five, and late-onset, appearing after the age of five. This may be a more appropriate division as growth velocity of the T1 to L5 spine peaks between birth and the age of five and because cardiopulmonary complications are more likely seen when onset occurs before the age of five. ${ }^{3}$ By these modern definitions, the diagnosis of AIS presently falls within the category of late-onset idiopathic scoliosis (LIS). Given this fact, care for patients with AIS may be guided by modern standards described for either AIS or LIS.

\section{Epidemiology}

Adolescent Idiopathic Scoliosis is a relatively common disorder with an overall prevalence of $0.47 \%-5.2 \%$ found in studies observing children of various nationalities. ${ }^{4}$ Of the three classic periods of onset for idiopathic scoliosis, adolescent onset is by far the most common accounting for as many as $90 \%$ of diagnosed cases. ${ }^{5}$ In some nations, such as Germany and the United Kingdom, infantile onset was very common in the past and accounted for almost $50 \%$ of cases of idiopathic scoliosis. However, this has dropped to $4 \%$ or lower with the adoption of prone infant-positioning practices. ${ }^{6}$

AIS is significantly more common in female children with an average female to male prevalence ratio of $2.1: 1$ to $2.36: 1$ based on data from large recent studies. ${ }^{7,8}$ The exact prevalence of curve types also varies by gender, but for both males and females thoracic curves are the most common (44.06\% in boys, $49.10 \%$ in girls) followed by thoracolumbar/lumbar ( $49.55 \%$ in boys, $36.09 \%$ in girls) and the less common double (4.26\% in boys, $11.10 \%$ in girls) and double thoracic curves $\left(2.14 \%\right.$ in boys, $3.71 \%$ in girls). ${ }^{4}$ In addition to curve type, gender has also been shown to have an impact on the observed severity of scoliotic curves. Several studies have shown that AIS deformities with Cobb angles of $20^{\circ}$ or larger are significantly more common in females than males such that the age matched prevalence ratio may be greater than $8: 1 .^{9,10}$

\section{Etiology}

While there are many established causes of scoliosis including rickets, vertebral malformations, torticollis, and leg length discrepancies, by definition, idiopathic scoliosis refers to a lateral curvature that is presently of unknown cause. ${ }^{11}$ That being said, numerous potential etiologies for adolescent idiopathic scoliosis have been discussed in modern literature. Some of these include abnormalities in connective tissue structure, muscle physiology, neurologic and osseous development, and platelet chemistry as well as specific aberrations in hormone levels. ${ }^{12}$

\section{Connective tissue}

Supported by the presence of scoliosis in patients with connective tissue disorders such as Marfan syndrome, the idea that connective tissue irregularity may be an etiologic factor for AIS has received a significant amount of attention. Structural abnormalities of these tissues including decreased elastic fiber density and non-uniform 
fiber distribution in the ligamentum flavum have been identified in patients with AIS. ${ }^{13}$ However, it is now generally accepted that these changes develop secondary to the structural forces experienced by those studied tissues as a result of the deformity. ${ }^{14}$

\section{Muscle physiology}

Underlying muscular pathology has also been studied as a potential soft tissue cause of AIS. Research in the past has revealed microscopic muscular irregularities in patients with AIS such as a significantly lower percentage of type 1 fibers in the concave side erector spinae muscles when compared to those muscles of age and gender matched controls. ${ }^{15}$ Much like the implicated connective tissue irregularities, it is difficult to show that these are causative abnormalities and not changes occurring secondary to the deformity. ${ }^{16}$.

\section{Neuro-osseous development}

As the result of efforts to identify possible growth derangements related to AIS, several etiological theories regarding uneven development of the spinal column and spinal cord have been suggested..$^{17}$ In 2001 Robert Porter posed the hypothesis that vertebrae may deform and rotate during growth to protect a relatively short spinal cord. Studies using MRI and multiplanar reconstruction have revealed reduced spinal cord to vertebral column ratios in AIS patients and have supported the notion that a tethering effect on the spinal cord may initiate curve development. ${ }^{18}$

\section{Platelet chemistry}

Platelet abnormalities of intracellular calcium concentration have been implicated in the etiopathogenesis of AIS, but studies on this topic have yielded inconsistent results. Recently it has been found that there is an underlying abnormality in platelet Ca2+ATPase in patients with AIS. The observation of this platelet channelopathy along with a similar abnormality in osteoblasts in these patients has led to the suggestion that a systemic defect in cell differentiation may be involved in the etiology of AIS. ${ }^{19}$

\section{Hormone levels}

Dysfunction of the melatonin signaling pathway has also been suggested as a possible cause of AIS. Animal studies have shown that an absence of melatonin leads to the development of scoliosis. Furthermore, the examination of tissue samples taken from patients with AIS during spine surgery has revealed impaired melatonin signaling in osteoblasts. ${ }^{20}$ While these are interesting findings, the mechanism by which melatonin irregularities might promote AIS has yet to be elucidated.

\section{Consensus on associated abnormalities}

Despite the observed presence of the aforementioned abnormalities in patients with AIS, systematic review has concluded that there is insufficient strong evidence to show a consistent pattern of occurrence between many implicated conditions and AIS. ${ }^{21}$ Furthermore, in studies that do suggest a definite relationship between associated abnormalities and AIS, it is often not established that these are true etiological factors rather than secondary changes due to the underlying pathophysiology of the disorder. ${ }^{16}$

\section{Modern studies of etiology}

Due to the persistent challenge of proving a causal relationship between associated pathologies and AIS, genetic research has been carried out in recent years in order to explore the etiology of this deformity on a molecular level. As there is an apparent difference in AIS prevalence by gender, it has been suggested that the etiology may have roots in an X chromosome abnormality. While pursuing this notion, one large study looking at families with multiple individuals diagnosed with AIS found a locus on the X chromosome that may be involved in development of the deformity. ${ }^{22}$ Despite findings such as this, some authors feel that an X-chromosome abnormality is an unlikely primary cause of AIS based on the fact that X-linked disorders are typically more common in males than females. ${ }^{23}$

With respect to autosomal chromosomes, recent genome wide association studies have identified several specific loci that warrant further investigation. The $3 \mathrm{p} 26.3$ locus is of particular interest as it contains candidate genes for AIS susceptibility such as CHL $1 .{ }^{24}$ Other loci that may contain susceptibility genes may be found on chromosomes $6,9,16$, and $17 .{ }^{25}$

Given the wide number of implicated genes and the multitude of associated abnormalities, it is presently believed that the etiology of AIS is multifactorial in nature. ${ }^{16}$ While identifying the true cause of AIS has proven to be a challenging task, as our understanding of this deformity improves, more effective screening and detection techniques may be developed to allow for earlier management and the prevention of large curve development.

\section{Physiology}

\section{Anatomy}

While often characterized by the lateral curvature of the spine, adolescent idiopathic scoliosis is a three dimensional deformity involving multiplaner vertebral changes (Figure 1). In addition to vertebral rotation in the coronal plane producing the lateral curvature, changes also occur in the sagittal and transverse planes. In the sagittal plane, a relative lordosis is generally observed in involved vertebrae of the thoracic spine. In the transverse plane, vertebrae are rotated such that the spinous processes point toward the curves concavity. The subsequent change in costal positioning that this produces in the thoracic spine results in the rotational prominence that is typically observed when a patient with AIS assumes the leaning forward position. ${ }^{26}$

A variety of different curve patterns may be seen in patients with AIS including thoracic curves, thoracolumbar curves, double curves, and double thoracic curves. Of these deformities, thoracic curves are the most commonly observed. ${ }^{4}$ When these $\mathrm{C}$ shaped curves are identified at the thoracic level, they are typically right convex in nature. Similar thoracic deformity may be noted in S shaped double curves with the opposite convexity seen in the thoracolumbar curve. In this $\mathrm{S}$ pattern, the lumbar curvature is often greater than the thoracic curvature, so cosmetic abnormalities such as rotational rib prominence may be less obvious. ${ }^{27}$

\section{Long term complications}

Numerous complications of untreated AIS have been explored in the study of this spinal deformity. Back pain, compromised selfimage, physical disability, pulmonary symptoms and early mortality are among the most commonly discussed undesirable outcomes. ${ }^{28}$

Back pain: Reports of back pain on long term follow up for patients with untreated idiopathic scoliosis are highly variable with rates as high as $90 \%$ or as low as $40 \%$ based upon the given study. Despite such variability, most authors have found that patients with untreated idiopathic scoliosis more frequently experience back pain than individuals in the general population. ${ }^{29}$ Operative treatment has been shown in past studies to decrease back pain for treated patients 
compared to those with untreated AIS, but not to decrease it to that of a control population..$^{30} \mathrm{~A}$ more recent study by Danielsson and Nachemson found that back pain in patients 20 years following spinal arthrodesis was only minimally greater than that in controls. ${ }^{31}$ In the future, there may be value in carrying out a long term study of modern minimally invasive surgical techniques to determine if back pain for the operated population may finally approach that of the population without AIS

A)
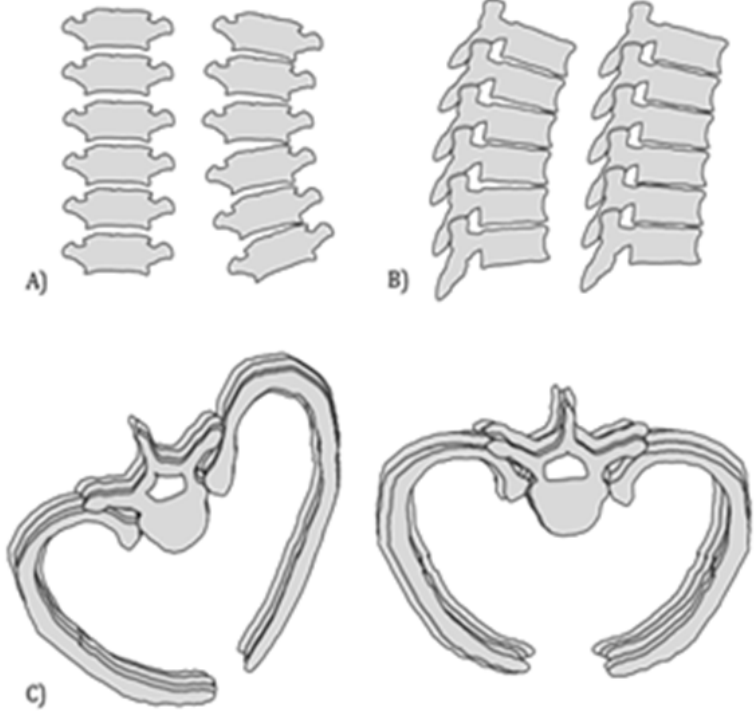

Figure I Changes in vertebral rotation observed in adolescent idiopathic scoliosis.

a) Coronal plane: Left: Normal thoracic vertebrae. Right: Coronal rotation produces the characteristic lateral curvature of AIS.

b) Sagittal plane: Left: Normal thoracic vertebrae. Right: Sagittal changes result in a relative lordosis in the thoracic spine (kyphotic angle less than 20 degrees).

c) Transverse plane: Left: Normal thoracic vertebrae. Right: Rotation in the transverse plane produces the rib hump easily observed in the forward bending position.

Compromised self-image: Similar to back pain, compromised selfimage has generally been found to be a true complication of untreated AIS. In a study in 1988 that addressed this specific issue, $56 \%$ of patients reported that they were self-conscious or depressed about their body shape. This was worse in patients with curves over $90^{\circ}$ as $77 \%$ of those individuals were distressed by their body habitus. ${ }^{32}$ In a 2003 study of various AIS complications, the negative long term impact of this deformity on self-image was further supported by the finding that average body satisfaction was lower for the AIS population compared to the control population on all of the scales that were utilized. ${ }^{33}$

Physical disability: Unlike back pain and compromised self-image, there is not a general consensus regarding physical disability as a long term complication of untreated AIS. While some authors have suggested that this deformity leads to a decreased ability to carry out physical activities ${ }^{34}$ others have found no significant difference between patients with AIS and controls in their ability to perform activities such as lifting heavy objects, walking long distances, or sitting for extended periods of time. Furthermore, while disability due to back pain was identified as a cause of reduced working hours in Weinstein's recent study, this decrease in work activity was not shown to be significantly greater for the AIS population compared to the control population. ${ }^{33}$
Pulmonary symptoms: Pulmonary symptoms are another topic of some debate in the natural history of AIS. While it is generally accepted that large curves may lead to restrictive lung disease and shortness of breath, ${ }^{29}$ some studies have suggested that there is not a significant increase in pulmonary symptoms for patients with curves of less than $50^{\circ} .{ }^{33}$ However, it has recently been found that, while basal respiratory function may be spared, individuals with curves averaging $32.8^{\circ}$ demonstrate lower ventilatory efficiency and generally lower physical performance than controls during exercise testing. ${ }^{35}$

Mortality: Despite the presence of historical papers that indicate an increased mortality rate of more than twice that of the age matched general population for patients with idiopathic scoliosis, ${ }^{36}$ it is currently believed that such results were skewed by the inclusion of patients with congenital scoliosis and that those mortality rates do not hold true for all types of scoliosis. ${ }^{29}$ Pehrsson's long term follow up study supports this point as it was found that mortality was significantly increased for patients 40-50 years of age with untreated infantile and juvenile idiopathic scoliosis, but that this was not the case for patients with adolescent idiopathic scoliosis ${ }^{37}$ In a more recent 50 year follow up study, it was stated that there is insufficient evidence to link untreated AIS with increased rates of mortality in general, or from cardiac or pulmonary conditions caused by the deformity. ${ }^{33}$

While AIS may not increase average mortality, it is important to note that some severe curvatures can be responsible for life threatening complications. For instance, it has been found that patients with a curve angle greater than $110^{\circ}$ and a vital capacity below $40 \%$ of predicted value are at increased risk for death due to respiratory failure..$^{38}$ Other studies that have commented on patients dying of scoliosis related complications have all reported that those individuals had curves greater than $100^{\circ}$ with the majority of the curves being greater than $140^{\circ} .{ }^{28}$

\section{Curve progression}

Given the relationship between curve size and long term sequelae, curve progression has long been a topic of interest in the study of AIS. In 1983 Weinstein and Ponseti found that curves with Cobb angles of less than $30^{\circ}$ at skeletal maturity generally did not to progress. Thoracic curves between $40^{\circ}$ and $50^{\circ}$ progressed by approximately $1^{\circ}$ per year through adulthood and curves between $50^{\circ}$ and $75^{\circ}$ showed even greater progression. ${ }^{39}$ In 1986 Ascani addressed curve progression by specific type and suggested that thoracic curves progress the most followed by lumbar, thoracolumbar, and then double curves. ${ }^{40}$

A more recent study designed to test the Sanders Skeletal Maturity Staging System revealed general findings similar to Weinstein's as none of the patients with initial curves of $15^{\circ}$ or less had curve progression while most of the patients with curves of $40^{\circ}$ or greater prior to skeletal maturity went on to have curve progression to greater than $50^{\circ}$. This study further divided the probability progression by Sanders skeletal maturity stage. It stated that the percentage of patients with curve progression after observation of an initial curve of $30^{\circ}$ at maturity stages 1,3 , and 4 was $67 \%, 43 \%$ and $27 \%$ respectively. ${ }^{41}$ These findings highlight the significant variability in AIS prognosis and the importance of skeletal maturity in the understanding of AIS physiology.

\section{Diagnosis}

\section{Presentation}

Patients with Adolescent idiopathic Scoliosis often present with cosmetic concerns such as uneven shoulders, hip asymmetry or a rib prominence recognized by the patient or a family member. ${ }^{42}$ Back 
pain may also be a presenting complaint for patients with AIS. This is more likely for individuals who are at an advanced stage of skeletal maturity, older than fifteen, or postmenarchal. ${ }^{43}$

\section{Physical exam}

Prior to examination of spinal curve characteristics in a patient with suspected AIS, a thorough neurological exam should be carried out. This may reveal an underlying intraspinal pathology such as syringomyelia, which has been found to be associated with the absence of superficial abdominal reflexes on the convex side of the curve. ${ }^{44}$ Such neurological findings may be an indication for further investigation with magnetic resonance imaging.

When examining the patients back, characteristics that should be assessed include shoulder obliquity, waist crease asymmetry, sagittal balance, coronal decompensation, and trunk rotation. ${ }^{45}$ Most of these features may be observed with the patient standing, but trunk rotation is best viewed with the patient in the bending forward position described by Adams in 1865. While the USPSTF does not recommend performing this as a screening test for AIS, ${ }^{46}$ it is useful in a focused spinal examination as it accentuates the degree of rotation observed in the surface of the back. ${ }^{47}$ With the patient in the bending forward position, the angle of trunk rotation may be estimated using a scoliometer, a small measuring device which is placed on the patient's back over the spinous process at the level of the curve apex. ${ }^{48}$

While the diagnosis of AIS may be apparent on physical exam, standard imaging studies are carried out in the assessment of these patients in order to quantify specific characteristics of their deformity

\section{Imaging studies}

Roentgenography: The X-ray imaging studies typically used in the diagnosis and assessment AIS include standing anterior-posterior (AP) or posterior-anterior (PA) views, sideward bending AP or PA views, and sagittal views. Coronal views should include the cervical spine and the pelvis in one image while sagittal views should span from the cranium to both femoral heads to facilitate various measurements of coronal and sagittal balance. ${ }^{49}$ Three significant measurements made on these images are the Cobb angle, the clavicle angle, and the sagittal balance (Figure 2).

Cobb angle: The Cobb angle is found on the AP view by drawing a line parallel to the superior endplate of the top vertebra and a line parallel to the inferior endplate of the bottom vertebra. Those terminal vertebrae are the most tilted and yield the greatest Cobb angle. Intersecting lines perpendicular to the endplates of the terminal vertebrae are drawn and the superior angle formed between them is measured as the Cobb angle. ${ }^{50} \mathrm{~A}$ diagnosis of AIS is confirmed when a lateral curvature with a Cobb angle of $10^{\circ}$ or greater is present on a standing coronal radiograph. ${ }^{51}$ Curvatures of non-structural nature may be differentiated from curvatures of structural nature on bending films as non-structural curves will bend out to less than $25^{\circ}$ while structural curves will remain equal to or greater than $25^{\circ} . .^{2}$

Clavicle angle: The clavicle angle is measured as the angle between a tangential line connecting the highest two points of each clavicle and an intersecting horizontal line. This measurement has been found to have a stronger correlation with shoulder balance after spinal arthrodesis than most other coronal plane measurements and may be useful in operative planning to achieve cosmetically satisfying results..$^{53}$

Sagittal balance: The sagittal balance is the distance between a plumb line dropped from the center of the $\mathrm{C} 7$ vertebral body and the posterior-superior aspect of the $\mathrm{S} 1$ vertebral body on the sagittal view. This is an important measurement as sagittal imbalance, defined as a $4 \mathrm{~cm}$ or greater deviation from neutral, has been identified as a cause of back pain in patients with spinal deformity. ${ }^{54}$ Given this observation, correction of sagittal imbalance should be viewed as a major goal in the treatment of AIS with the aim of improving long term quality of life. ${ }^{55}$
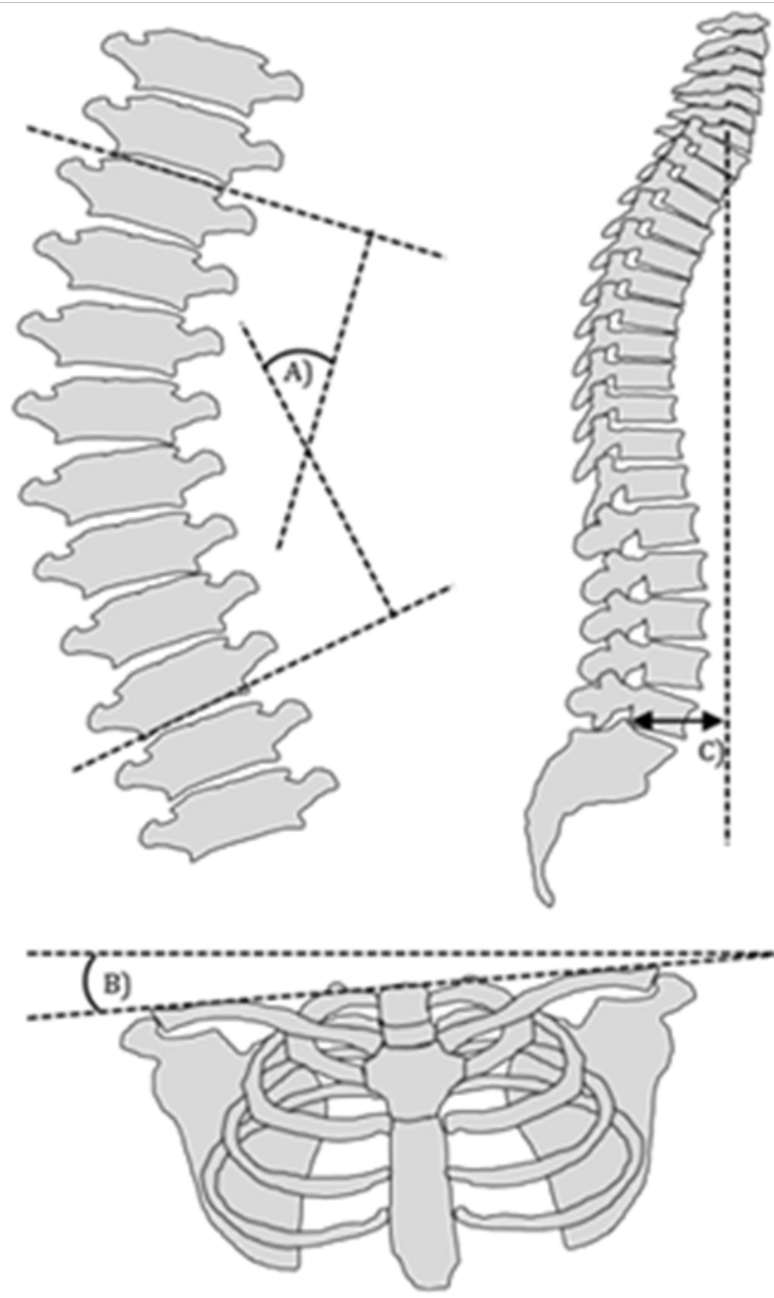

Figure 2 Radiographic measurements used in the assessment of AIS.
a) Cobb Angle
b) Clavicle Angle

c) Sagittal Balance

Magnetic resonance imaging: MRI is typically not used in the diagnosis of AIS. However, MRI may be useful in the detection of associated neurological deformities such as syringomyelia. The presence of these deformities in patients with presumed AIS has been shown to increase the risk for neurological injury during spinal arthrodesis procedures ${ }^{56} \mathrm{~A}$ recent study found that a combination of an atypical curve pattern and neurological findings had the greatest diagnostic yield as an indication for performing an MRI as central nervous system deformities were identified in $25 \%$ of patients with these findings. The best single indicator was an atypical curve pattern such as the absence of thoracic apical segment lordosis, while the worst single indicator was back pain as no patients with this symptom alone who received an MRI were found to have a central nervous system abnormality. ${ }^{57}$ In the absence of abnormal physical or neurological 
findings, MRI is generally not indicated as a screening test prior to arthrodesis since the diagnostic yield for neurological deformity in these scenarios is very low at less than $2 \%{ }^{58}$

\section{Determination of skeletal maturity}

As discussed under physiology, the probability of curve progression for patients with AIS is related to the Cobb angle of their spinal curvature and their degree of skeletal maturity. Thus, efforts are often made at the time of diagnosis to assess the patient's degree of skeletal maturity.

While indicators such as chronological age, menarchal status, and trends in height and weight may be used for a quick assessment of skeletal maturity, ${ }^{59}$ numerous radiographic techniques have been developed in the study of scoliosis to allow for more accurate estimation.

Risser sign: In 1958 Joseph Risser described a relationship between spinal skeletal maturity and ossification of the iliac crest apophysis. ${ }^{60}$ This relationship became the foundation for the present day Risser sign, a six stage system in which stage 0 is characterized by no ossification of the apophysis and signifies the beginning of the curve acceleration phase of spinal growth ${ }^{61}$ while stage 5 is characterized by complete fusion of the ossified apophysis to the iliac crest and signifies the cessation of vertebral growth. ${ }^{62}$

Since the advent of the Risser scale, several other techniques for skeletal assessment have been developed including the Oxford Method first described by Acheson in $1954,{ }^{63}$ the Greulich and Pyle method described in 1971, and the Tanner Whitehouse II method described in $1983 .{ }^{64}$

Sanders skeletal maturity scale: A modern system for the determination of skeletal maturity in patients with AIS was described by James Sanders in 2008. This radiographic method of classification divides skeletal maturity into eight stages determined by specific characteristics observed on anterior-posterior radiographs of the hand. These eight stages correspond with defined phases of spinal curve acceleration. Based upon the degree of spinal curvature observed at these stages, this classification system also suggests a given patient's risk of curve progression. ${ }^{65}$ In addition to having prognostic use at diagnosis, information such as this may be used to guide suggestions for bracing and other treatments.

\section{Management}

As discussed earlier, back pain, compromised self-image, physical disability, pulmonary symptoms and early mortality are all possible long term outcomes of scoliotic deformity. ${ }^{28}$ Unlike infantile idiopathic scoliosis, which resolves spontaneously in more than $70 \%$ of cases, ${ }^{66}$ the deformity in AIS generally does not improve without intervention. In order to avoid the complications that may be associated with AIS, some patients are candidates for non-operative or operative therapy.

\section{Non operative therapy}

The primary non-operative therapy suggested for the management of adolescent idiopathic scoliosis is the use of an orthotic brace. ${ }^{67}$

Indications: Current indication criteria for the use of bracing to treat adolescent idiopathic scoliosis include a patient age of 10 years or older, Risser stage of $0-2$, major curve angle of $25^{\circ}-40^{\circ}$, no prior treatment, and premenarchal or less than 1 year postmenarchal status for females. ${ }^{68}$ Some authors have recently suggested that these indications are be too broad and that bracing should be limited to patients at high risk for clinically significant curve progression as to avoid unnecessary treatment of individuals who will not benefit from that therapy. ${ }^{69}$

Types of braces: There are numerous types of orthoses used around the world in the treatment of AIS. In North America, the Boston brace is the most commonly prescribed orthoses brace. This is a rigid, back opening, polyethylene brace that is popular with patients for its low profile and relatively cosmetically acceptable design. ${ }^{70}$ Other rigid AIS orthoses used around the world include the Charleston, Cheneau, Lyon, Milwaukee, PASB, Providence, TLI and Wilmington braces. The SpineCor and TriaC are two non-rigid orthoses that have also been designed for the management of AIS. There is limited research on these relatively new devices, but cohort studies for each have indicated that they both significantly reduce curve progression. ${ }^{71,72}$

Bracing technique: Orthotic braces used for the management of AIS should be worn for as many hours of the day as tolerable. In general, it is suggested that the orthoses be worn for 22 hours per day from the time at which bracing is first indicated until the time at which the patient reaches skeletal maturity. ${ }^{73}$ This suggestion is supported by a recent multicenter prospective study which concluded that there is in fact a positive dose-response relationship between hours of brace wear and prevention of curve progression. That study found that more than $90 \%$ of patients who wore their brace for an average of 12.9 hours per day or more did not have curve progression past $50^{\circ}$ while patients who wore their brace for an average of less than 6 hours per day did have curve progression past $50^{\circ}{ }^{69}$

Outcomes: Systematic reviews have indicated that bracing is effective in preventing curves from progressing more than $6^{\circ}$, but these reviews have not shown that bracing significantly decreases the likelihood of treated patients going on to have surgery. ${ }^{74}$ However, recent studies suggest that those outcomes were negatively impacted by poor compliance and that the number needed to treat to prevent surgery in patients with AIS may be as low as three when braces are properly used. ${ }^{75}$ In addition to decreasing the need for surgery, bracing has been found to reduce the long term prevalence and severity of back pain in patients treated for AIS close to that of a normal control population. ${ }^{76}$

\section{Operative therapy}

The gold standard operative therapy for the management of Adolescent Idiopathic Scoliosis is segmental spinal instrumentation and fusion. ${ }^{77}$

Indications: Absolute indications for spinal arthrodesis are a topic for debate in the study of AIS. Traditional indications for surgery are a curve this greater than $45-50^{\circ}$ at skeletal maturity or a curve that is greater than $40-45^{\circ}$ degrees in a patient with $1-2$ years or more of growth remaining. ${ }^{78}$ While these may still be acceptable guidelines based on our understanding of the long term complications of large curves, the decision to perform arthrodesis should be made on an individual bases due to wide variation in physiology. For instance, spinal arthrodesis might not be necessary for a patient with a double curve in the $50^{\circ}$ range due to a balancing effect and a relatively normal cosmetic appearance. On the other hand, this surgery may be appropriate for a patient with a single thoracolumbar curve in $35-45^{\circ}$ range due to coronal decompensation and a relatively undesirable cosmetic appearance. ${ }^{79}$

Level selection: Once it has been found that surgery is indicated for a given patient, a decision must be made regarding which vertebrae to include in the instrumented fusion. Several classifications systems have been developed in order to offer guidance during this decision making process. 
Table I Primers for RT-PCR

\begin{tabular}{|c|c|c|c|c|}
\hline \multicolumn{5}{|l|}{ Curve Type } \\
\hline Lenke Type & $\begin{array}{l}\text { Proximal } \\
\text { Thoracic Curve }\end{array}$ & $\begin{array}{l}\text { Main Thoracic } \\
\text { Curve }\end{array}$ & $\begin{array}{l}\text { Thoracolumbar } \\
\text { /Lumbar curve }\end{array}$ & Curve Type \\
\hline I & Non-Structural & Structural (Major) & Non-Structural & Main Thoracic \\
\hline 2 & Structural & Structural (Major) & Non-Structural & Double Thoracic \\
\hline 3 & Non-Structural & Structural (Major) & Structural & Double Major \\
\hline 4 & Structural & Structural (Major) & Structural & Triple Major \\
\hline 5 & Non-Structural & Non-Structural & Structural (Major) & Thoracolumbar/ Lumbar \\
\hline 6 & Non-Structural & Structural & Structural (Major) & $\begin{array}{l}\text { Thoracolumbar/ Lumbar-Main } \\
\text { Thoracic }\end{array}$ \\
\hline \multicolumn{5}{|l|}{ Modifiers } \\
\hline $\begin{array}{l}\text { Lumbar Spine } \\
\text { Modifiers }\end{array}$ & \multirow{2}{*}{\multicolumn{2}{|c|}{$\begin{array}{l}\text { CSVL to Lumbar Apex } \\
\text { Vertebra Relationship } \\
\text { CSVL runs between } \\
\text { pedicles }\end{array}$}} & $\begin{array}{l}\text { T5-T I } 2 \text { Thoracic Sagittal } \\
\text { Profile Modifier }\end{array}$ & Angle of Kyphosis \\
\hline A & & & - (Hypokyphosis) & $<10^{\circ}$ \\
\hline B & $\begin{array}{l}\text { CSVL touches lateral } \\
\text { aspect of the vertebral body }\end{array}$ & & N (Normal kyphosis) & $10^{\circ}-40^{\circ}$ \\
\hline C & $\begin{array}{l}\text { CSVL completely } \\
\text { medical to vertebral body }\end{array}$ & & + (Hyperkyphosis) & $>40$ \\
\hline
\end{tabular}

King classification: In 1983 King and Moe described a system that divided scoliotic curves into five major types.

Type I curves are S shaped patterns in which both curves cross the mid line, but the lumbar curve is larger than the thoracic. Similarly, type II curves are $\mathrm{S}$ shaped patterns in which both curves cross the mid line, but the thoracic curve is larger than the lumbar. Type III curves have a pattern in which there is a thoracic curve which crosses the midline and a lumbar curve which does not. Type IV patterns are characterized by a single long thoracic curvature with L5 over the sacrum and L4 tilted into the curve while type $\mathrm{V}$ patterns are characterized by a double thoracic curvature with $\mathrm{T} 1$ tilted into the convexity.

Based on these different curve types, the following suggestions were made for vertebral fusion. For type I patterns, both curves should be fused to the lower vertebra, but no lower than L4. For type II patterns, the thoracic curve should be fused to the lower vertebra that is both neutral and stable or just to the most stable vertebra. For type III and IV patterns, fusion should include the measured thoracic curve, with the lower level of fusion ending at the first vertebra that is most closely bisected by the center sacral line. Finally, for type $\mathrm{V}$ patterns, both thoracic curves should be fused with the lower level of fusion ending at the first vertebra that is most closely bisected by the center sacral line. ${ }^{80}$

While the King classification system and its suggestions were widely accepted after its development, subsequent reviews of this method have found that it has relatively low inter-observer reliability and does not seem adequate to allow for surgical decision making based on reports made by other surgeons. ${ }^{81}$ Furthermore, this classification was designed to help determine the extent instrumentation with Harrington rods, and was not conceived with data regarding modern methods of segmental instrumentation. ${ }^{82}$

Lenke classification: In 2001 Lenke described a new AIS classification system designed to have a stronger inter-observer reliability than the King system and offer guidance for modern arthrodesis procedures. This system divided scoliotic deformity into six major types based on various combinations of curves. These curves were described by their location and structural nature. In accordance with the definitions established by the Scoliosis Research Society, a curve is thoracic if the apex occurs between $\mathrm{T} 2$ and the T11/12 disc, thoracolumbar if the apex is between T12 and L1, and lumbar if it is between the L1/ L2 disc and L4.$^{83}$ The thoracic curves may be further divided into proximal thoracic curves with the apex at $\mathrm{T} 3, \mathrm{~T} 4$, or $\mathrm{T} 5$, and main thoracic curves with the apex between T6 and the eleventh and the T11/12 disc. Any of these curves may be deemed structural if its Cobb angle remains equal to or greater than $25^{\circ}$ on side bending imaging.

In addition to the six major types, Lenke also described two modifiers to further characterize the three dimensional structure of scoliotic deformities. The lumbar modifier describes the relationship between the center sacral vertical line and the lumbar apex while the sagittal thoracic modifier describes the kyphotic angle of the thoracic spine between the T5 and T12 endplates. An overview of the Lenke classification system is presented in Table $1 .{ }^{52}$

Unlike the paper describing the King classification system, Lenke's 2001 paper does not list a specific set of guidelines to follow when making fusion level decisions based on each curve type. Since the publication of that paper, numerous suggestions have been made regarding the clinical application of the Lenke classification system. In a 2013 review, a fusion protocol was described that discussed fusion levels for all of the Lenke classifications. In addition to offering general guidelines such as the inclusion of all structural curves in the instrumentation, this review also made detailed suggestions regarding which upper and lower vertebrae to include in the fusion based on Lenke curve types and modifiers. ${ }^{84}$

Approach: In an international consensus statement published in 2014 a $96 \%$ majority declared that the posterior surgical approach was the optimal form of care when performing spinal arthrodesis in routine scenarios.$^{85}$ However, other techniques such as anterior open and anterior thoracoscopic approaches have also been advocated in modern literature. ${ }^{86}$ In a recent five year follow up study of patients who underwent anterior thoracoscopic spinal fusion it was found that these individuals were more likely to suffer from pseudarthrosis or hardware failure, but that they otherwise had similar outcomes to patients who underwent a posterior approach. Furthermore, it was noted the anterior thoracoscopic approach had several advantages including the cosmetic benefit of a smaller scar and the functional benefit of requiring fusion of fewer motion segments. ${ }^{77}$

Implants : The instrumentation involved in the treatment of AIS has evolved significantly over the last century. Various states of the 
art have come and passed working up from Hibbs's uninstrumented fusion, to Herrington's distraction rod, to Luque's sublaminar wire and rod technique, and now to modern day pedicle screw constructs. ${ }^{87}$ Recently, there has been some debate as to whether or not all pedicle screw constructs are the superior form instrumentation when compared to hybrid constructs which have screws in the lower vertebrae and hooks on the upper vertebrae. ${ }^{82}$ However, multiple studies in the last decade have found that all pedicle screw constructs to be superior with respect to outcomes including coronal correction, scoliometer measurement, and post-operative pulmonary function. ${ }^{88,89}$

In addition to an evolution in implant type, there has also been a notable change in implant materials over the years. While Harrington Rods were stainless steel devices, most modern constructs are composed primarily of titanium pedicle screws and titanium rods. ${ }^{85}$ For spinal fusion of more severe curves, cobalt-chromium rods are now also commonly used in as they have been found to exert higher corrective forces than stainless steel rods while still maintaining their shape. ${ }^{90}$

Intraoperative monitoring: In the practice of modern spinal arthrodesis, several technologies have been implemented to improve the safety of vertebral instrumentation. Two of these technologies are spinal cord monitoring and computer assisted navigation.

Spinal cord monitoring is a widely used technique in which leads are placed to measure somatosensory-evoked potentials or motorevoked potentials traveling through the spinal cord. In the event of intraoperative neurological injury, changes in the measurements of these potentials should alert the surgeon to begin corrective efforts. ${ }^{91}$

Unlike spinal cord monitoring, computer assisted navigation is not routinely used by most surgeons when performing spinal arthrodesis. ${ }^{85}$ Despite its limited utilization, recent studies have suggested that computer assisted navigation results in significantly higher accuracy of pedicle screw placement when compared to traditional methods. ${ }^{92}$ Furthermore, while the acquisition and maintenance of equipment such as an $\mathrm{O}$-arm is relatively expensive, reductions in re-operation costs associated with its use have been found to offset those expenses at institutions where there is a high volume of instrumented spinal procedures. ${ }^{93}$ Interestingly, even with improved accuracy and reduced re-operation rates, multiple systematic reviews have failed to show a reduction in neurological complications when using computer assisted navigation. ${ }^{92,94}$ Until more data is published that indicates improved functional outcomes, the overall clinical benefit of these technologies may remain up for debate.

Outcomes: Given the constant evolution of spinal arthrodesis techniques for the treatment of AIS, it is challenging to determine the long term outcomes for these procedures. Twenty year follow up studies performed in the past have found favorable results with respect to Cobb angles and pulmonary function ${ }^{95}$ and less favorable results with respect to social limitation and physical function. ${ }^{96}$ However, those studies examined the effects of treatments performed over 40 years ago, so their conclusions might not apply to modern procedures.

In an effort to address the impact of modern surgical methods, a 2011 study looked at a group of AIS patients who had undergone spinal arthrodesis in the nineteen nineties. While this study did find that the cohort of patients had some acceleration of L5-S1 disc degeneration, the outcomes were otherwise positive as it revealed that most of the patients had minimal pain or functional impairment over a decade after their surgery. ${ }^{97}$ In the future, similar research will have to be carried out to determine the long term benefits of today's spinal fusion techniques.

\section{Conclusion}

There is a vast amount of literature available for review regarding adolescent idiopathic scoliosis. The associated knowledge base is profoundly organic, with an ever changing understanding of AIS such that the very definition of the deformity itself has evolved in recent years. With constant progress being made toward a proper understanding of the molecular and genetic roots of this disease, it is conceivable that preventative measures may someday be developed to stop AIS from ever developing. In the meantime, armed with a thorough understanding of the physiology, diagnosis, and management of this disorder, modern physicians should be able to greatly reduce the prevalence of scoliotic deformity and its associated complications in the adult population.

\section{Acknowledgments}

None.

\section{Conflicts of interest}

None.

\section{References}

1. Goldstein LA, Waugh TR. Classification and terminology of scoliosis. Clin Orthop Relat Res. 1973 ;(93):10-22.

2. J James. Idiopathic Scoliosis The prognosis diagnosis and operative indications related to curve patterns and the age at onset. $J$ Bone Joint Surg Br. 1954;36-B(1):36-49.

3. Gillingham BL, Fan RA, Akbarnia BA. Early onset idiopathic scoliosis. $J$ Am Acad Orthop Surg. 2006;14(2):101-112.

4. Konieczny MR, Senyurt H, Krauspe R. Epidemiology of adolescent idiopathic scoliosis. J Child Orthop. 2013;7(1):3-9.

5. McMaster MJ. Infantile idiopathic scoliosis: Can it be prevented. Am J Bone Joint Surg Br. 2007;65(5):612-617.

6. Lincoln TL. Infantile idiopathic scoliosis. Am J Orthop. 2007;36(11):586-590.

7. Soucacos PN, Soucacos PK, Zacharis KC, et al. School-Screening for Scoliosis: A prospective epidemiological study in northwestern and central greece. J Bone Jt Surg. 1997;79A(10):1498-1503.

8. Suh SW, Modi HN, Yang JH, et al. Idiopathic scoliosis in Korean schoolchildren: a prospective screening study of over 1 million children. Eur Spine J. 2011;20(7):1087-1094.

9. Morais T, Bernier M, Turcotte F. Age- and Sex-specific Prevalence of Scoliosis and the Value of School Screening Programs. Am J Public Health. 1985;75(12):377-380.

10. Ueno M, Takaso M, Nakazawa T, et al. 5-year epidemiological study on the prevalence rate of idiopathic scoliosis in Tokyo: School screening of more than 250000 children. J Orthop Sci. 2011;16(1):1-6.

11. A Whitman. Observations on the corrective and operative treatment of structural scoliosis. Arch Surg. 1922;5(3):578-630.

12. Dayer R, Haumont T, Belaieff W, et al. Idiopathic scoliosis: etiological concepts and hypotheses. J Child Orthop. 2013;7(1):11-16.

13. Hadley-Miller N, Mims B, Milewicz DM. The potential role of the elastic fiber system in adolescent idiopathic scoliosis. J Bone Joint Surg Am. 1994;76(8):1193-1206.

14. Lowe TG, Edgar M, Margulies JY, et al. Etiology of idiopathic scoliosis: current trends in research. J Bone Joint Surg Am. 2000;82-A(8):1157-1168.

15. Bylund P, Jansson E, Dahlberg E, et al. Muscle fiber types in thoracic erector spinae muscles Fiber types in idiopathic and other forms of scoliosis. Clin Orthop Relat Res. 1987;214:222- 228. 
16. Cheung KM, Wang T, Qiu GX, et al. Recent advances in the aetiology of adolescent idiopathic scoliosis. Int Orthop. 2008;32(6):729-734.

17. Chu WC, Lam WM, Ng BK, et al. Relative shortening and functional tethering of spinal cord in adolescent scoliosis - Result of asynchronous neuro-osseous growth summary of an electronic focus group debate of the IBSE. Scoliosis. 2008;3(8):E1-E24.

18. Chu WC, Lam WW, Chan YL, et al. Relative shortening and functional tethering of spinal cord in adolescent idiopathic scoliosis?: study with multiplanar reformat magnetic resonance imaging and somatosensory evoked potential. Spine (Phila Pa 1976). 2006;31(1):E19-E25.

19. R Bredoux ,E Corvazier, S Dally, et al. Human platelet Ca2+-ATPases: New markers of cell differentiation as illustrated in idiopathic scoliosis. Platelets. 2006;17(6):421-433.

20. Moreau A, Wang DS, Forget S, et al. Melatonin signaling dysfunction in adolescent idiopathic scoliosis. Spine (Phila Pa 1976). 2004;29(16):1772-1781.

21. Schlösser TP, van der Heijden GJ, Versteeg AL, et al. How 'Idiopathic' Is Adolescent Idiopathic Scoliosis? A Systematic Review on Associated Abnormalities. PLoS One. 2014;9(5):e97461.

22. Justice CM, Miller NH, Marosy B, et al. Familial idiopathic scoliosis: evidence of an X-linked susceptibility locus. Spine (Phila Pa 1976). 2003;28(6):589-594.

23. Raggio CL. Sexual Dimorphism in Adolescent Idiopathic Scoliosis. Orthop Clin North Am. 2006;37(4):555-558.

24. Sharma S, Gao X, Londono D, et al. Genome-wide association studies of adolescent idiopathic scoliosis suggest candidate susceptibility genes. Hum Mol Genet. 2011;20(7):1456-1466.

25. Miller NH, Justice CM, Marosy B, et al. Identification of Candidate Regions for Familial Idiopathic Scoliosis. Spine (Phila Pa 1976). 2005;30(10):1181-1187.

26. Dickson RA. Idiopathic scoliosis: foundation for physiological treatment. Ann R Coll Surg Engl. 1987;69(3):89-96.

27. Hefti F. Pathogenesis and biomechanics of adolescent idiopathic scoliosis (AIS). J Child Orthop. 2013;7(1):17-24.

28. Asher MA, Burton DC. Adolescent idiopathic scoliosis: natural history and long term treatment effects. Scoliosis. 2006;1(1):2.

29. Danielsson AJ. Natural history of adolescent idiopathic scoliosis: a tool for guidance in decision of surgery of curves above $50^{\circ} \mathrm{c} . J$ Child Orthop. 2013;7(1):37-41.

30. Dickson JH, Mirkovic S, Noble PC, et al. results of operative treatment of idiopathic scoliosis in adults. J Bone Jt Surg Am. 1995;77(4):513-523.

31. Danielsson AJ, Nachemson AL. Back pain and function 23 years after fusion for adolescent idiopathic scoliosis: a case-control study-part II. Spine (Phila Pa 1976). 2003;28(18):E373-E383.

32. Edgar MA, Mehta MH. Long-term follow-up of fused and unfused idiopathic scoliosis. J Bone Joint Surg Br. 1988;70(5):712-716.

33. Weinstein SL, Dolan LA, Spratt KF, et al. Health and function of patients with untreated idiopathic scoliosis: a 50-year natural history study. Jama. 2003;289(5):559-567.

34. Goldberg MS, Mayo NE, Poitras B, et al. The Ste-Justine Adolescent Idiopathic Scoliosis Cohort Study Part II: Perception of health self and body image and participation in physical activities. Spine (Phila Pa 1976). 1994;19(14):1562-1572.

35. Barrios C, Pérez-Encinas C, Maruenda JI, et al. Significant ventilatory functional restriction in adolescents with mild or moderate scoliosis during maximal exercise tolerance test. Spine (Phila Pa 1976). 2005;30(14):1610-1615.

36. Nilsonne U, Lundgren KD. Long-Term Prognosis in Idiopathic Scoliosis. Acta Orthop. 1968;39(4):456-465.
37. Pehrsson K, Larsson S, Oden A, et al. Long-term follow-up of patients with untreated scoliosis A study of mortality causes of death and symptoms. Spine (Phila Pa 1976). 1992;17(9):1091-1096.

38. Pehrsson $\mathrm{K}$, Bake $\mathrm{B}$, Larsson $\mathrm{S}$ et al. Lung function in adult idiopathic scoliosis: a 20 year follow up. Thorax. 1991;46(7):474-478.

39. Weinstein SL, Ponseti IV Curve progression in idiopathic scoliosis. $J$ Bone Joint Surg Am. 1983;65(4):447-455.

40. Ascani E, Bartolozzi P, Logroscino CA et al. Natural history of untreated idiopathic scoliosis after skeletal maturity. Spine (Phila Pa 1976). 1986;11(8):784-789.

41. Sitoula P, Verma K, Holmes L et al. Prediction of Curve Progression in Idiopathic Scoliosis: Validation of the Sanders Skeletal Maturity Staging System. Spine (Phila Pa 1976). 2015;40(13):1006-1013.

42. F Altaf, A Gibson, Z Dannawi et al. Adolescent idiopathic scoliosis. BMJ 346(f2508): 1-7.

43. Ramirez N, Johnston CE, Browne RH The prevalence of back pain in children who have idiopathic scoliosis. J Bone Joint Surg Am. 1997;79(3):364-368.

44. Saifuddin A, Tucker S, Taylor BA et al. Prevalence and clinical significance of superficial abdominal reflex abnormalities in idiopathic scoliosis. Eur Spine J. 2005;14(9):849-853.

45. D Studer Clinical investigation and imaging. $J$ Child Orthop. 2013;7(1):29-35.

46. A Berg, J Allan, N Calonge et al. Screening for Idiopathic Scoliosis: Recommendation Statement. USPSTF.2004;1-3.

47. Stokes IA, Moreland MS Measurement of the shape of the surface of the back in patients with scoliosis The standing and forward-bending positions. J Bone Joint Surg Am. 1987;69(2):203-211.

48. Bunnell WP An objective criterion for scoliosis screening. J Bone Joint Surg Am. 1984;66(9):1381-1387.

49. Malfair D, Flemming AK, Dvorak MF et al. Radiographic Evaluation of Scoliosis: Review. Am J Roentgenol 194(3 Suppl): 2010;8-S22.

50. J Cobb Outline for the study of scoliosis. Am Acad Orthop Surg Inst Course Lect. 1948;5:261-275.

51. Van Goethem J, Van Campenhout A, van den Hauwe L et al.Scoliosis. Neuroimaging Clin N Am. 2007;17(1):105-115.

52. Lenke LG, Betz RR, Harms Jet al. Adolescent Idiopathic Scoliosis. J Bone Jt Surg. 2001;83-A (8):1169-1181.

53. Kuklo TR, Lenke LG, Graham EJ et al. Correlation of radiographic clinical and patient assessment of shoulder balance following fusion versus nonfusion of the proximal thoracic curve in adolescent idiopathic scoliosis. Spine (Phila Pa 1976). 2002;27(18):2013-2020.

54. Bridwell KH, Lewis SJ, Lenke LG et al. Pedicle subtraction osteotomy for the treatment of fixed sagittal imbalance. J bone Joint Surg Am. 2003;85-A(3):454-463.

55. Glassman SD, Berven S, Bridwell K, et al. Correlation of radiographic parameters and clinical symptoms in adult scoliosis. Spine (Phila Pa 1976). 2005;30(6):682-688.

56. Noordeen MH, Taylor BA, Edgar MA. Syringomyelia A potential risk factor in scoliosis surgery. Spine (Phila Pa 1976). 1994;19(12):1406-1409.

57. Davids JR, Chamberlin E, Blackhurst DW. Indications for Magnetic Resonance Imaging in Presumed Adolescent Idiopathic Scoliosis. $J$ Bone Joint Surg. 2004;86-A(10):2187-2195.

58. Do T, Fras C, Burke S, et al. Clinical value of routine preoperative magnetic resonance imaging in adolescent idiopathic scoliosis: a prospective study of three hundred and twenty-seven patients. $J$ Bone Joint Surgery Am. 2001;83-A(4):577-579. 
59. Sanders JO, Browne RH, McConnell SJ, et al. Maturity assessment and curve progression in girls with idiopathic scoliosis. J Bone Joint Surg Am. 2007;89(1):64-73.

60. Joseph CR. The Classic: The Iliac Apophysis: An Invaluable Sign in the Management of Scoliosis. Clin Orthop Relat Res. 2010;468(3):646-653.

61. Nault ML, Parent S, Phan P, et al. A Modified Risser Grading System Predicts the Curve Acceleration Phase of Female Adolescent Idiopathic Scoliosis. s. 2010;92(5):1073-1081.

62. Hacquebord JH, Leopold SS. In Brief: The Risser Classification: A Classic Tool for the Clinician Treating Adolescent Idiopathic Scoliosis Clin Orthop Relat Res. 2012;470(8):2335-2338.

63. Acheson RM. A method of assessing skeletal maturity from radiographs; a report from the Oxford child health survey. J Anat. 1954;88(Pt4):498-508.

64. Bull RK, Edwards PD, Kemp PM, et al. Bone age assessment : a large scale comparison of the Greulich and Pyle and Tanner and Whitehouse (TW2) methods Bone age assessment : a large scale comparison of the Greulich and Pyle and Tanner and Whitehouse (TW2) methods. Arch Dis Child. 1999;81(2):172-173.

65. Sanders JO, Khoury JG, Kishan S, et al. Predicting Scoliosis Progression from Skeletal Maturity: A Simplified Classification During Adolescence. J Bone Joint Surg Am. 2008;90(3):540-553.

66. Thompson SK, Bentley G. Prognosis in Infantile Idiopathic Scoliosis. $J$ Bone Joint Surg Am. 1980;62-B(2):151-154.

67. Sponseller PD. Bracing for adolescent idiopathic scoliosis in practice today. J Pediatr Orthop. 2011;31(1 Supp1):S53-S60.

68. Richards BS, Bernstein RM, D'Amato CR, et al. Standardization of criteria for adolescent idiopathic scoliosis brace studies: SRS Committee on Bracing and No operative Management. Spine (Phila Pa 1976) 30(18): 2068-2075; discussion. 2005;2076-2077.

69. Weinstein SL, Dolan LA, Wright JG, Dobbs MB (2013) Effects of Bracing in Adolescents with Idiopathic Scoliosis. N Engl J Med 369(16): 1512-1521

70. Zaina F, De Mauroy JC, Grivas T, et al. Bracing for scoliosis in 2014 state of the art. Eur J Phys Rehabil Med. 201450(1):93-110.

71. A Circo C, Coillard, C Rivard. Effectiveness of the SpineCor brace based on the standardized criteria proposed by the SRS for adolescent idiopathic scoliosis - up to date results. Scoliosis. 2009;4(Suppl 2):054

72. Gerben JB, Albert GV, Gert N. Clinical effect of continuous corrective force delivery in the non-operative treatment of idiopathic scoliosis: a prospective cohort study of the triac-brace. Eur Spine J. 2008;17(2):231-239.

73. Dietrich S, Timo Y. Bracing in adolescent idiopathic scoliosis. $J$ Child Orthop. 2013;7(1):51-55.

74. Sanders JO, Newton PO, Browne RH, et al. Bracing in adolescent idiopathic scoliosis, surrogate outcomes, and the number needed to treat. J Pediatr Orthop. 2012;32(Suppl 2):S153-S157.

75. Sanders JO, Newton PO, Browne RH, et al. Bracing for idiopathic scoliosis: how many patients require treatment to prevent one surgery? $J$ Bone Joint Surg Am. 2014;96(8):649-653.

76. Danielsson AJ, Nachemson AL. Back pain and function 22 years after brace treatment for adolescent idiopathic scoliosis: A case-control study - Part I. Spine (Phila Pa 1976). 2003;28(18):2078-2085.

77. Newton PO, Upasani VV, Lhamby J, et al. Surgical Treatment of Main Thoracic Scoliosis with Thoracoscopic Anterior Instrumentation: A FiveYear Follow-up Study. J Bone Joint Surg Am. 2008;90(10):2077-2089.

78. Edward L, John S. Idiopathic Scoliosis: New Instrumentation for Surgical Management. J Am Acad Orthop Surg. 1994;2(1):67- 78.
79. Bridwell KH. Surgical treatment of idiopathic adolescent scoliosis. Spine (Phila Pa 1976). 1999;24(24):2607-2616.

80. King HA, Moe JH, Bradford DS, et al. The selection of fusion levels in thoracic idiopathic scoliosis. J Bone Joint Surg Am. 1983;65(9):1302-1313.

81. Cummings RJ, Loveless EA, Campbell J, et al. Interobserver reliability and intraobserver reproducibility of the system of King et al for the classification of adolescent idiopathic scoliosis. J Bone Joint Surg Am. 1998;80(8):1107-1111.

82. Cuartas E, Rasouli A, O'Brien M, et al. Use of all-pedicle-screw constructs in the treatment of adolescent idiopathic scoliosis. $J$ Am Acad Orthop Surg. 2009;17(9):550-561.

83. Lowe T, Berven SH, Schwab FJ, et al. The SRS classification for adult spinal deformity: building on the King/Moe and Lenke classification systems. Spine (Phila Pa 1976). 2006;31(19 Suppl):S119-S125.

84. Trobisch PD, Ducoffe AR, Lonner BS, et al. Choosing fusion levels in adolescent idiopathic scoliosis. J Am Acad Orthop Surg. 2013;21(9):519-528.

85. de Kleuver M, Lewis SJ, Germscheid NM, et al. Optimal surgical care for adolescent idiopathic scoliosis: an international consensus. Eur Spine J. 2014;23(12):2603-2618.

86. Sucato DJ. Thoracoscopic anterior instrumentation and fusion for idiopathic scoliosis. J Am Acad Orthop Surg. 2003;11(4):221-227.

87. Carol CH. A brief overview of 100 years of history of surgical treatment for adolescent idiopathic scoliosis. J Child Orthop. 2013;7(1):57-62.

88. Kim YJ, Lenke LG, Kim J, et al. Comparative analysis of pedicle screw versus hybrid instrumentation in posterior spinal fusion of adolescent idiopathic scoliosis. Spine (Phila Pa 1976). 2006;31(3):291-298.

89. Luhmann SJ, Lenke LG, Erickson M, et al. Correction of moderate (<70 degrees) Lenke 1A and 2A curve patterns: comparison of hybrid and all-pedicle screw systems at 2-year follow-up. J Pediatr Orthop. 2012;32(3):253-258.

90. Lamerain M, Bachy M, Delpont M, et al. CoCr rods provide better frontal correction of adolescent idiopathic scoliosis treated by allpedicle screw fixation. Eur Spine J. 2014;23(6):1190-1196.

91. Pahys JM, Guille JT, D'Andrea LP, et al. Neurologic injury in the surgical treatment of idiopathic scoliosis: guidelines for assessment and management. J Am Acad Orthop Surg. 2009;17(7):426-434.

92. Tian NF, Huang QS, Zhou P, et al. Pedicle screw insertion accuracy with different assisted methods: a systematic review and meta-analysis of comparative studies. Eur Spine J. 2011;20(6):846-859.

93. Nicolas D, Charles F, Juliet B, et al. Economic Evaluation of Intraoperative Cone Beam CT-Based Navigation for the Placement of Spinal Pedicle Screws: A Patient Level Cost Effectiveness Analysis. Spine J. 2015;05:A012.

94. Verma R, Krishan S, Haendlmayer K, et al. Functional outcome of computer-assisted spinal pedicle screw placement: a systematic review and meta-analysis of 23 studies including 5992 pedicle screws. Eur Spine J. 2010;19(3):370-375.

95. Pehrsson K, Danielsson A, Nachemson A. Pulmonary function in adolescent idiopathic scoliosis: a 25 year follow up after surgery or start of brace treatment. Thorax. 2001;56(5):388-393.

96. Danielsson AJ, Wiklund I, Pehrsson K, et al. Health-related quality of life in patients with adolescent idiopathic scoliosis: a matched followup at least 20 years after treatment with brace or surgery. Eur Spine J. 2001;10(4):278-288.

97. Green DW, Lawhorne TW, Widmann RF, et al. Long-Term Magnetic Resonance Imaging Follow-up Demonstrates Minimal Transitional Level Lumbar Disc Degeneration After Posterior Spine Fusion for Adolescent Idiopathic Scoliosis. Spine (Phila Pa 1976). 2011;36(23):1948-1954. 